\title{
Construction of Whole Genome Microarrays, and Expression Analysis of Desulfovibrio vulgaris cells in Metal-Reducing Conditions (Uranium and Chromium)
}

\author{
Fields, Matthew W. \\ Miami University
}

\begin{abstract}
RESULTS TO DATE: One of the major goals of the project is to construct whole-genomoe microarrays for Desulfovibrio vulgaris. Previous whole-genome microarrays constructed at ORNL have been PCRamplimer based, and we wanted to re-evaluate the type of microarrays being built because oligonucleotide probes have several advantages. Microarrays have been generally constructed with two types of probes, PCR-generated probes that typically range in size between 200 and $2000 \mathrm{bp}$, and oligonucleotide probes with typical size of 20-70 nt. Producing PCR product-based DNA arrays can be a time-consuming procedure that includes PCR primer design, amplification, size verification, product purification, and product quantification. Also, some ORFs are difficult to amplify and thus the construction of comprehensive arrays can be a challenge. Recently, to alleviate some of the problems associated with PCR product-based microarrays, oligonucleotide microarrays that contain probes longer than 40 nt have been evaluated and used for whole genome expression studies. These microarrays should have higher specificity and are easy to construct, and can thus provide an important alternative approach to monitor gene expression. However, due to the smaller probe size, it is expected that the detection sensitivity of oligonucleotide arrays will be lower than PCR product-based probes. In addition, various studies reported in the literature use oligonucleotide probes that range from $20 \mathrm{nt}$ to $70 \mathrm{nt}$, but a direct comparison of the performance between oligonucleotide probes of different lengths has not been previously reported. First, in order to experimentally establish the criteria for designing gene-specific oligonucleotide probes, an
\end{abstract} oligonucleotide array was constructed that contained perfect match (PM) and mismatch (MM) probes (50mers and 70mers) based upon 4 genes. The effects of probe-target identity, continuous stretch, mismatch position, and hybridization free energy on specificity were examined. Little hybridization was observed at a probe-target identity of $<85 \%$ for both 50 mer and 70 mer probes. 33 to $48 \%$ of the PM signal intensities were detected at a probe-target identity of $94 \%$ for 50 mer oligonucleotides, and 43 to $55 \%$ for 70 mer probes at a probe-target identity of $96 \%$. When the effects of sequence identity and continuous stretch were considered independently, a stretch probe ( $>15$ bases) contributed an additional $9 \%$ of the PM signal intensity compared to a non-stretch probe ( $<15$ bases) at the same identity level. Cross-hybridization increased as the length of continuous stretch increased. A 35-base stretch for 50mer probes or a 50 -base stretch for 70 mer probes had approximately $55 \%$ of the PM signal. Mismatches should be as close to the middle position of an oligonucleotide probe as possible to minimize crosshybridization. Little cross-hybridization was observed for probes with a minimal binding free energy greater than $-30 \mathrm{kcal} / \mathrm{mol}$ for $50 \mathrm{mer}$ probes or $-40 \mathrm{kcal} / \mathrm{mol}$ for 70 mer probes. Based on the experimental results, a set of criteria were suggested for the design of gene-specific and group-specific oligonucleotide probes, and these criteria should provide valuable information for the development of new software and algorithms for microarray-based studies. Secondly, in order to empirically determine the effect of probe length on signal intensities, microarrays with oligonucleotides of different lengths were used to monitor gene expression at a whole genome level. To determine what length of oligonucleotide is a better alternative to PCR-generated probes, the performance of oligonucleotide probes was systematically compared to that of their PCR-generated counterparts for 96 genes from Shewanella oneidensis MR-1 in terms of overall signal intensity, numbers of detected genes, specificity, sensitivity and differential gene expression under experimental conditions. Hybridizations conducted at $420 \mathrm{C}, 450 \mathrm{C}, 50 \mathrm{oC}$, and $60 \mathrm{oC}$ indicated that good sensitivities were obtained at $450 \mathrm{C}$ for oligonucleotide probes in the presence of $50 \%$ formamide, under which conditions specific signals were detected by both PCR and oligonucleotide probes. Signal intensities increased as the length of oligonucleotide probes increased, and the 70mer oligonucleotide probes produced similar signal intensities and detected a similar number of ORFs compared to the PCR probes. CDNA, 70mer, 60mer and 50mer arrays had detection sensitivities at 5.0, 25, 100 and $100 \mathrm{ng}$ of genomic DNA, or an approximately equivalent of $1.9 \times 106,9.2 \times 106,3.7 \times 107$ 
and $3.7 \times 107$ copies, respectively when the array was hybridized with genomic DNA. To evaluate differential gene expression under experimental conditions, S. oneidensis MR-1 cells were exposed to low or high $\mathrm{pH}$ conditions for 30 and $60 \mathrm{~min}$, and the transcriptional profiling detected by oligonucleotide probes (50mer, 60mer, and 70mer) was closely correlated with that detected by the PCR probes. The results demonstrated that 70 mer oligonucleotides can achieve the most comparable performance with PCR-generated probes. Desulfovibrio vulgaris has a genome size of $3.6 \mathrm{Mb}$ and 3584 predicted ORFs. The whole-genome microarrays of $D$. vulgaris have been constructed using 70 mer oligonucleotides. All ORFs in the genome were represented with 3471 (97.1\%) unique probes and 103 (2.9\%) non-specific probes. In order to evaluate the performance of the experimental microarrays, growth conditions were selected that were expected to give significant hybridization differences for different sets of genes. The initial evaluations were performed using $D$. vulgaris cells grown at logarithmic and stationary phases with lactate and sulfate as electron donor and acceptor, respectively. Transcriptional analysis of $D$. vulgaris cells sampled during logarithmic phase growth indicated that $25 \%$ of annotated ORFs were up-regulated and $3 \%$ of annotated ORFs were down-regulated compared to stationary phase cells. The up-regulated genes included ORFs predicted to be involved with acyl chain biosynthesis, amino acid ABC transporter, translational initiation factors, and ribosomal proteins. In the stationary phase growth cells, the two most up-regulated ORFs (70-fold) were annotated as a carboxynorspermidine decarboxylase and a 2C-methylD-erythritol-2,4-cyclodiphosphate (MECDP) synthase. Spermidines are polyamines that are typically abundant in rapidly dividing cells and are essential growth factors in eukaryotic organisms. Polyamines are thought to stabilize DNA by the association of the amino groups with the phosphate residues of DNA and can also enhance tRNA and ribosome stability. Several of the highly up-regulated ORFs were annotated as conserved hypothetical proteins. Interestingly, an ORF that was predicted to contain a flocculin repeat domain was almost 9-fold up-regulated in stationary phase cells compared to cells in the logarithmic phase. The flocculin domain is commonly observed in fungi, and is thought to play a role during flocculation (non-sexual aggregation of single-cell microorganisms). These preliminary results have identified possible responses of $D$. vulgaris cells to stationary phase growth and suggest that polyamine production as well as cell aggregation and/or extracellular polymer production are responses of $D$. vulgaris during stationary phase. The initial microarray results indicate that the recently produced oligonucleotide microarrays are functional. We are currently optimizing growth conditions in order to study D. vulgaris under different growth conditions. Currently, growth conditions and medium are being evaluated for transcriptomic characterization with respect to stasis-regulated genes and heavy metal responsive genes. The elucidation of growth-phase dependent gene expression is crucial for a general understanding of growth physiology, but will also be crucial for data interpretation of stress-responsive genes (e.g., chromium, uranium). We have been coordinating (e.g., medium composition and growth conditions) with the VIMSS:GTL project ?Rapid deduction of stress response pathways in metal/radionuclide reducing bacteria? in order to facilitate data interpretation, minimize overlap, and maximize productivity. We recently cultivated $D$. vulgaris in a defined medium and sampled biomass over time for approximately $70 \mathrm{~h}$ to characterize the shifts in gene expression as cells entered stationary phase growth from logarithmic phase growth. Preliminary experiments indicate that RNA was obtained and labeled, and good hybridization signals were observed. In addition, protein, carbohydrate, sulfate, lactate, and acetate levels are being determined. We are presently determining the optimal methods and conditions for growth in the presence of $\mathrm{Cr}(\mathrm{VI})$. Preliminary data suggests that $0.05 \mathrm{mM} \mathrm{Cr}(\mathrm{VI})$ but not $0.01 \mathrm{mM} \mathrm{Cr}(\mathrm{VI})$ decreased growth in a defined medium with lactate and sulfate. In addition, D. vulgaris grew well in a defined, minimal medium designed to be analogous to FRC groundwater.

DELIVERABLES: Manuscripts

Z. He, L. Wu, X. Li, M.W. Fields and J. Zhou. Empirical establishment of oligonucleotide probe design criteria. Appl. Environ. Microbiol. (in review)

Z. He, L. Wu, M.W. Fields and J. Zhou. Comparison of microarrays with different probe sizes for monitoring gene expression. Appl. Environ. Microbiol. (in review) Abstracts and Presentations 
Z. He, Q. He, L.Wu, J.D. Wall, J. Zhou, M.W. Fields. 2004. Construction and evaluation of D. vulgaris whole-genome oligonucleotide microarrays and comparison of cells in exponential and stationary phase growth. American Society for Microbiology, 104th General Meeting, New Orleans, LA

X. Li, Z. He, C. W. Schadt, S. C. Chong, J. Liebich, T. J. Gentry, M. W. Fields and J. Zhou. 2004.

Development of CommOligo to select unique and group specific probes for oligonucleotide microarrays using global alignment. American Society for Microbiology, 104th General Meeting, New Orleans, LA

M.W. Fields. 2004. Characterization of physiological responses of metal- and sulfate-reducing bacteria using genomics. presentatioin at Medical College of Ohio, Toledo, $\mathrm{OH}$ 